\title{
Specifying polarimetric tolerances of a high-resolution imaging multiple- species atmospheric profiler (HiMAP)
}

Juliana Mae Richter, Russell Chipman, Brian Daugherty, David J. Diner, Annmarie Eldering, et al.

Juliana Mae Richter, Russell Chipman, Brian Daugherty, David J. Diner, Annmarie Eldering, Jason J. Hyon, Meredith Kupinski, Jessica L. Neu, Dejian $\mathrm{Fu}$, "Specifying polarimetric tolerances of a high-resolution imaging multiplespecies atmospheric profiler (HiMAP)," Proc. SPIE 10925, Photonic Instrumentation Engineering VI, 109250F (22 May 2019); doi: $10.1117 / 12.2510750$

SPIE. Event: SPIE OPTO, 2019, San Francisco, California, United States 


\title{
Specifying Polarimetric Tolerances of a High-resolution Imaging Multiple-species Atmospheric Profiler (HiMAP)
}

\author{
Juliana Richter ${ }^{\mathrm{a}}$, Russell Chipman ${ }^{\mathrm{a}}$, Brian Daugherty ${ }^{\mathrm{a}, \mathrm{b}}$, David J. Diner ${ }^{\mathrm{c}}$, Annmarie Eldering ${ }^{\mathrm{c}}$, \\ Jason J. Hyon ${ }^{c}$, Meredith Kupisnki ${ }^{\mathrm{a}}$, Jessica L. Neuc ${ }^{\mathrm{c}}$, Dejian Fu ${ }^{\mathrm{c}}$ \\ ${ }^{a}$ University of Arizona, Optical Sciences, 1630 E. University Blvd. Tucson, AZ, U.S.A., 85719 \\ ${ }^{\mathrm{b}}$ Airy Optics, 4961 E Oakmont Dr, Tucson, AZ, U.S.A., 85718 \\ ${ }^{\mathrm{c}}$ Jet Propulsion Lab, 4800 Oak Grove Dr, Pasadena, CA, U.S.A., 91109
}

\begin{abstract}
The High-resolution Imaging Multiple-species Atmospheric Profiler (HiMAP) is an ultraviolet imaging spectro-polarimeter in development at the Jet Propulsion Laboratory for measuring $\mathrm{O}_{3}$ and $\mathrm{NO}_{2}$ concentrations in the troposphere from an airborne platform or satellite. In this paper: (1) the HiMAP design is illustrated and modeled using 3D polarization ray tracing calculus, (2) the dependency between the condition number of the systems polarization measurement matrix and properties of individual optical components is used as a method for tolerancing, and (3) the polarimeter capabilities of manufacturable thin film designs of polarizing and non-polarizing beam splitters is explored using numerical methods. The condition number of an optical system is calculated from a polarization ray tracing (PRT) matrix model of the polarimeter. Deviations of the condition number are calculated for non-ideal polarization elements and coatings to understand component and alignment tolerances.
\end{abstract}

Keywords: polarization, remote sensing, spectroscopy, tolerance analysis.

\section{Introduction}

Air quality is a growing priority within the atmospheric composition and applied science community. In particular, near-surface gaseous pollutants (ozone and nitrogen dioxide) and aerosols have been identified by NASA Decadal Surveys. ${ }^{1-4}$ It is estimated that ozone $\left(\mathrm{O}_{3}\right)$ destroys crops that would otherwise feed 94 million people each year. ${ }^{5}$ Nitrogen dioxide $\left(\mathrm{NO}_{2}\right)$ is an $\mathrm{O}_{3}$ precursor and a major contributor to respiratory disease. Because these gases are present throughout the atmospheric column, quantification of their global health and environmental impacts requires a major advance in the stateof-the-measurement of profiling their concentrations in the near-surface layer $(0-2 \mathrm{~km})$. Improvement in spatial resolution is also required in order to map spatial variability at the neighborhood (intra-urban) scale.

The High-resolution Imaging Multiple-species Atmospheric Profiler (HiMAP) is an ultraviolet imaging spectro-polarimeter in development at the Jet Propulsion Laboratory for measuring $\mathrm{O}_{3}$ and $\mathrm{NO}_{2}$ concentrations in the troposphere from an airborne platform or satellite. HiMAP enables the quantification of near-surface pollutants by using a combination of high- spectral resolution and multiangular viewing polarimetry over a continuous spectral region to finely sample these trace gas concentrations in the vertical dimension. Additional measurement requirements include global observations, high-spatial resolution, and high sensitivity as specified by the air quality community. 25 These requirements place stringent demands on acquiring spectrally resolved $(0.5 \mathrm{~nm}$ resolution), high-accuracy polarimetry (0.005 in degree of linear polarization (DoLP)) and imaging over a broad FOV (60) with an integration time of 0.5 second. HiMAP will enable these advanced measurement capabilities for the

Photonic Instrumentation Engineering VI, edited by Yakov G. Soskind,

Proc. of SPIE Vol. 10925, 109250F · C 2019 SPIE · CCC code:

0277-786X/19/\$18 - doi: $10.1117 / 12.2510750$

Proc. of SPIE Vol. 10925 109250F-1 
first time through spectrally resolved, multi-angle polarimetry in the UV-Visible. The measurement approach is also scalable to the Near Infrared $(680-780 \mathrm{~nm})$ to enable aerosol profiling within the oxygen absorption bands. The performance of a polarimeter concept for HiMAP, or the efficiency of polarization state separation, is quantified using the L2 condition number. ${ }^{6}$ The condition number is calculated directly from a polarization model of an optical system.

This paper is in six sections. Section 1 outlines the HiMAP polarimeter configuration and operation. Section 2 explains the application and design goals of the HiMAP polarimeter. Section 3 explains the model polarimeter. Section 4 discusses polarimetric tolerancing for the retarders and thin film coating designs of a polarizing beam splitter (PBS) and non-polarizing beam splitter (NPBS). Section 5 uses the results of condition number analysis when setting tolerance bounds of the polarimeter. The tolerancing methods outlined in this paper are discussed in the context of a linear Stokes polarimeter and can be generalized to other polarimeters. Section 6 concludes this paper, reviewing polarimeter tolerancing methods and expands on their applications.

Deviations of the condition number are tracked as individual parameters (reflectance, transmission, retardance, fast-axis, diattenuation) are varied to study sensitivities within an optical design. To illustrate the capabilities of this method to tolerance the components of an optical system with polarimetric qualities, this paper employs the example of a polarimeter.

Changes to the condition number reveal that the orientation of the quarter-wave plate (QWP) causes larger errors than the QWP retardance. This occurs because errors in the retardance self-correct since these optics are double-pass in HiMAP. However, errors in the orientation cause a stunted rotation of the s-state into the p-state, resulting in linear states measured as elliptical states. The waveplate parameter with the greatest sensitivity is the orientation of the half-wave plate (HWP), where $\pm 5^{\circ}$ increases the condition number by $23 \%$.

\section{Application and Design Goals}

HiMAP combines high spectral resolution and multi-angular viewing polarimetry to finely sample gaseous pollutants and aerosols in the vertical dimension. These requirements place stringent demands on acquiring spectrally resolved $(0.5 \mathrm{~nm}$ resolution), high accuracy polarimetric ( 0.005 in DoLP), and imaging over a broad field of view $\left(60^{\circ}\right)$.

HiMAP incorporates a wide field of view, spectrally resolved, multi-angular polarimetric measurements within a compact system to enable gaseous pollutant $\left(\mathrm{O}_{3}, \mathrm{NO}_{2}\right)$ and aerosol profiling. Spectrally resolved polarization signals have been proven using a JPL ground-based spectropolarimeter, ${ }^{7}$ but that technology is not suitable for compact flight instruments. Follow-on analysis indicates that a degree of freedom for signal (DOFS) of 0.7 to 1.0 of near surface gaseous pollutants could be achieved through spectrally resolved $(0.5 \mathrm{~nm})$, accurate polarimetric (0.005 in DoLP) measurements at multiple viewing angles, while the traditional single viewing-angle, radiance-only measurements provide a DOFS of 0.1 to 0.2 in the near surface layer. Additional measurement requirements include global observations, high spatial resolution, and high sensitivity as specified by the air quality community. ${ }^{8-11}$ These analyses guide the design requirements on spectral resolution and polarimetric accuracy. Note that an Observation System Simulation Experiment (OSSE) for HiMAP was conducted using JPLs MUlti-SpEctra, MUlti-SpEcies, MUlti-Sensors (MUSES) algorithm which is a validated algorithm, ${ }^{12-16}$ applied in the 
data production of satellite missions for atmospheric composition.

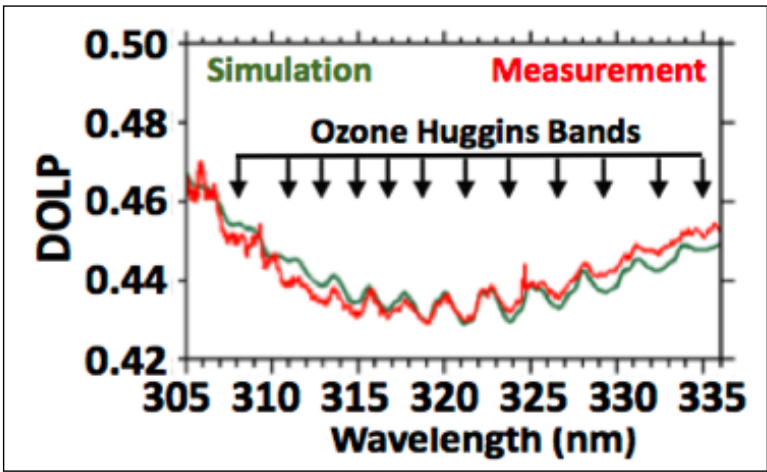

(a)

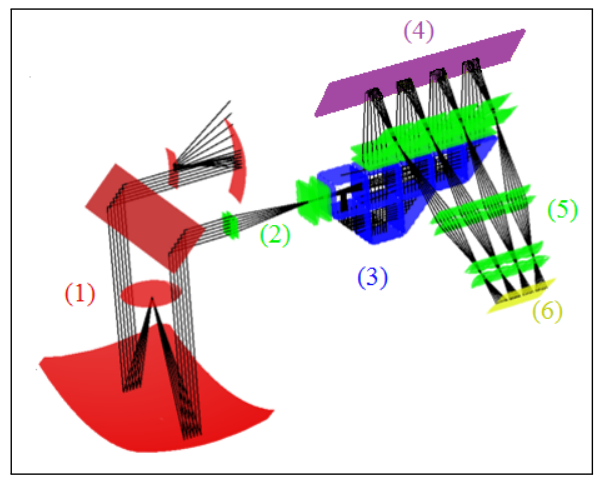

(b)

Fig 1 (a) Measured (red) DoLP of $\mathrm{O}_{3}$ from the rooftop of a JPL building and simulated (green curve) DoLP of $\mathrm{O}_{3}$. (b) Optical design of HiMAP instrument using Polaris-M polarization ray tracing program. The imaging polarimetry spectrometer (IPS) has a compact design of $6.0 \mathrm{~cm} 12.0 \mathrm{~cm} 18.0 \mathrm{~cm} \mathrm{(2U)} \mathrm{and} \mathrm{an} \mathrm{estimated} \mathrm{mass} \mathrm{of} 1,950 \mathrm{~g}$.

Conventional space spectrometers with nadir or whisk broom observations cannot accurately retrieve concentration profiles of tropospheric pollutants to obtain accurate near-surface information. However, by adding a DoLP capability in each wavelength bin and recording the same scene at multiple viewing angles, the vertical profile information content is increased. For instance, polarization measurements are greatly helpful for $\mathrm{O}_{3}$ retrievals because sunlight in the peaks and valleys of the $\mathrm{O}_{3}$ Huggins bands $(305-335 \mathrm{~nm})$ penetrates to different altitudes and therefore imprints the depolarization effects of each layer in the backscattered radiance. This spectrally varying polarization signature could be inverted using a vector radiative transfer model to obtain the concentration profiles. Additional vertical profile information could be retrieved by viewing at multiple angles along the orbital track because the weighted contribution to the total radiance from each layer varies by viewing angle.

HiMAP, for which the imaging polarimetry spectrometer (IPS) is the enabling optical subsystem, combines wide swath (field of view of $60^{\circ}$, or a swath width of $1,250 \mathrm{~km}$ in a low Earth orbit [LEO] of $700 \mathrm{~km}$ ), high accuracy linear polarization $(0.005)$, broad spectral range $(300-500 \mathrm{~nm})$, high spectral resolution $(0.5 \mathrm{~nm})$, and multiple along-track viewing angles $\left( \pm 70^{\circ}\right)$ to meet the precision, accuracy, and spatial resolution (global survey mode: $4 \times 4 \mathrm{~km}^{2}$; regional zoom-in mode: $1 \times 1 \mathrm{~km}^{2}$ ) requirements specified by community white papers submitted to the 2017 Earth Sciences Decadal Survey ${ }^{8-11}$ The unique capabilities of HiMAP include being: (1) the only approach that can quantify global near-surface $\mathrm{O}_{3}$ and one of its primary source gases, $\mathrm{NO}_{2}$, together (essential to quantifying the pollutants oxidation pathways), enabled by spectrally resolved polarimetry measurements over the continuous UV-Vis spectral region; (2) a compact solution (form factor: 6U/12U for suborbital/orbital missions) to deliver 3D maps of pollutants.

HiMAP contains six main optical components: (1) Cassegrain telescope, (2) relay optics, (3) division of amplitude polarimeter (DoAP), (4) grating, (5) imaging lens array (ILA), and (6) a detector. A telescope images light scattered by the atmosphere onto a thin slit, which serves as a field-stop. The light then enters the DoAP, which divides the light into four optical paths, or channels, according to 
the angle of linear polarization (AoLP). Relay optics then image light onto the detector using a lenslet array. Many division of amplitude polarimeters use multiple detectors. The HiMAP design enables the measurement of four polarization orientations using only a single focal plane array (FPA). This architecture permits synchronized readout of the polarimetric measurements in half the volume.

\section{Analysis of the HiMAP Polarimeter}

HiMAP images are acquired in a whisk broom scan. In a given instance, the horizontal position in each channel is related to the object-space field angle. Light from all four channels is spread along the vertical direction according to wavelength. Figure 3 shows the detector partitioned into four segments. A thin slit at the intermediate image plane between the telescope and the relay optics limits the vertical field to 2 pixels and the horizontal field to 512 pixels on the detector surface.

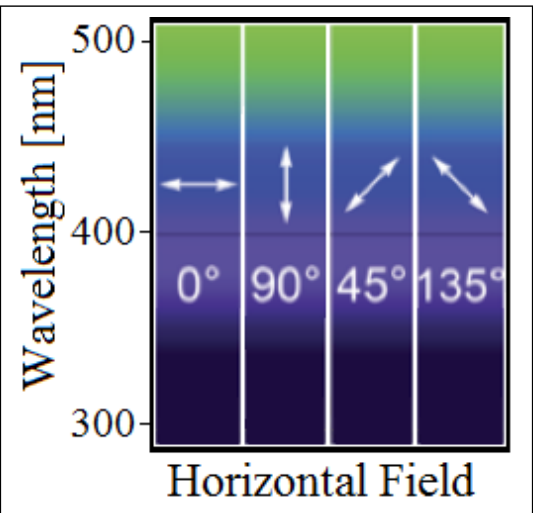

Fig 2 The detector is partitioned into four sections for each of the polarization channels. Light is dispersed in the vertical direction, while the slit is imaged in the horizontal direction. Each pixel corresponds to distinct AoLP, wavelength, and field position properties that can be attributed to radiance measurements.

The DoAP is a prism subsystem that separates incident light into four channels according to the AoLP: $0^{\circ}, 90^{\circ}, 45^{\circ}$, and $135^{\circ}$ (Fig. 2). The DoAP rotates the orientation of these linear polarization states so that they exit the prism with the same propagation vector, polarized in the plane of incidence $\left(90^{\circ}\right)$, and illuminate four separate areas of the grating. The ILA images four chromatically dispersed strips onto a single FPA, see Fig. 3. The sum and differences of radiance measurements from orthogonally polarized channels yields linear Stokes parameters,

$$
\mathbf{S}=\left[\begin{array}{c}
I \\
Q \\
U
\end{array}\right]=\left[\begin{array}{c}
I_{0^{\circ}}+I_{90^{\circ}} \\
I_{0^{\circ}}-I_{90^{\circ}} \\
I_{45^{\circ}}-I_{135^{\circ}}
\end{array}\right]
$$

where $I_{\theta}$ is the total radiance polarized in the $\theta$ direction (see Fig 3). The Stokes vector, $\mathbf{S}$, components have units of radiance. $I$ is the total radiance of the beam and $Q$ and $U$ are the radiance in each linear orthogonal polarization state. Boldface is used to differentiate vector-valued quantities. The circular term of the Stokes vector is ignored because the Earth's atmosphere presents negligible optical 
activity. ${ }^{17}$ The DoLP is a unit-less term, calculated from the Stokes parameters,

$$
\operatorname{DoLP}(\lambda, \phi)=\frac{\sqrt{Q(\lambda, \phi)^{2}+U(\lambda, \phi)^{2}}}{I(\lambda, \phi)},
$$

for each wavelength $\lambda$ and field angle $\phi$. A polarimeter that can measure variations in DoLP to within \pm 0.005 is needed to detect the presence of $\mathrm{O}_{3} .{ }^{8}$ The accuracy of the DoLP is dependent on the ability of the polarimeter to efficiently separate polarization states. The efficiency of polarimeter designs can be quantified using the L2 condition number., ${ }^{6,18}$ The condition number is sensitive to changes in the polarization measurement matrix ${ }^{19}$ due to: beamsplitter thin film designs, overall light loss, and ghost reflections. Polarization ray tracing software available from Airy Optics, Polaris-M, is used to compute the cumulative HiMAP polarization measurement matrix, and the linear contribution from each component. This analysis makes it possible to study system performance ${ }^{20}$ by relating the tolerances of each component to the condition number. ${ }^{18}$

\subsection{Polarimetric Modeling of System Components}

The HiMAP polarimeter divides light into four laterally-spaced optical paths. Figure 3 shows the DoAP fused-silica prism assembly. The incident polarization state, at a given field angle and wavelength, is related to the flux of each of the four paths, see Table 2.

Innovations of DoAP include: (a) double-pass thin film polarizing beam splitters (PBS) used for the first time in a DoAP, with four analyzed polarizations exiting in the same polarization state $\left(90^{\circ}\right.$ out of the plane), thus illuminating four separate areas of the grating with analyzed polarization states all transformed into one state to match the maximum diffraction efficiency; (b) ruggedized solid optic design that delivers twice the etendu per volume, in comparison to conventional air-spaced designs; (c) first simultaneous measurement of four polarized UV-Vis spectral images in remote sensing; and (d) polarizing and non-polarizing coating designs for wide spectral coverage and wide field of view (FOV: $60^{\circ}$ ).

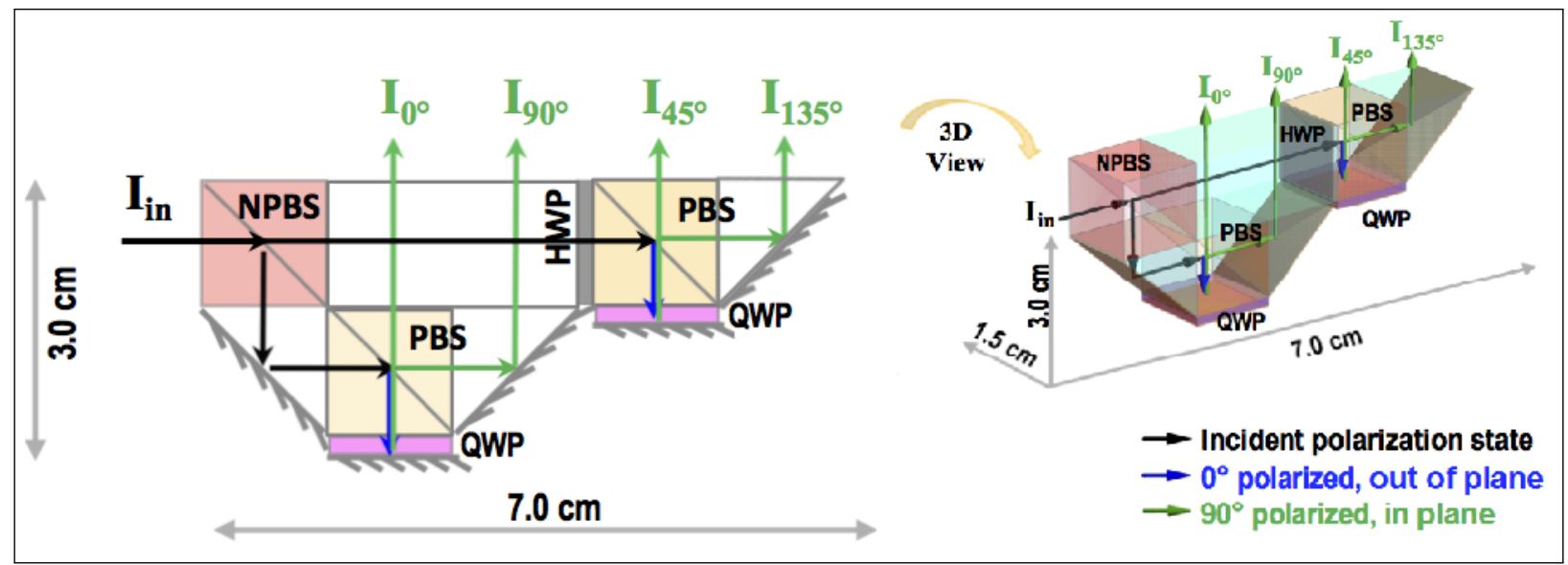

Fig 3 Right and isotropic views of the DoAP. Key features of the design are: same $k_{\text {out }}$ vectors, same exiting polarization state, and equal optical path length of the four channels (5-cubes). 
The DoAP (design shown in Figure 3) operates on incident-converging spherical wavefronts that outputs four spatially separated beams analyzing the flux, $I$, and linear polarizations, $Q$ and $U$. The NPBS ideally provides two beams with an unmodified polarization state. The transmitted NPBS state is rotated by $45^{\circ}$ with a HWP. Each pair of NPBS outputs are then analyzed by thin film polarizing beam splitters (PBS), one analyzing $0^{\circ}$ and $90^{\circ}$, the other $45^{\circ}$ and $135^{\circ}$; thus no light is lost in absorptive polarizers. To bring the beams out in the same direction, the downward propagating beams (blue), polarized at $0^{\circ}$, are converted to right circularly polarized light by a QWP, reflected from a mirror into left circularly polarized light, and then converted to $90^{\circ}$ (in the plane of the page) in the second pass through the QWP. These beams are now p-polarized at the PBS and thus transmit upwards and out of the polarimeter. This step ensures the four beams exit the DoAP in the same polarization state $\left(90^{\circ}\right.$ polarized, out of plane), though their flux indicates the four polarization components $\left(0^{\circ}, 45^{\circ}\right.$, $90^{\circ}$, and $135^{\circ}$ ) of the incident light. The QWP and mirror combinations in the DoAP's $0^{\circ}$ and $45^{\circ}$ paths provides a configuration that equalizes the path lengths through the four DoAP channels; all four beams image in the same plane with pupils in a shared plane.

Diffraction gratings can have large differences in diffraction efficiency between transverse-electric (TE) and transverse-magnetic (TM) polarizations, and these changes can vary rapidly with wavelength. Since the four beams exit the DoAP in the same polarization state, the grating efficiency differences do not compromise the polarimetric accuracy, ${ }^{21}$ and all four beams polarization states have been aligned for maximum grating efficiency, to improve SNR.

\section{Polarimetric Tolerancing}

The polarization ray tracing model integrates all DoAP surfaces and coatings to analyze polarization transformations and wavefront aberrations. The transmission and polarization properties of coated surfaces are modeled using polarization ray trace matrices (3D Jones matrices), ${ }^{19} \mathbf{J}$,to describe polarization state transformations in three-dimensional space using Jones notation extended to $3 \times 3$ matrices.

$$
\begin{aligned}
& \mathbf{J}_{Q W P}=\left(\begin{array}{ccc}
\frac{1}{\sqrt{2}} & 0 & \frac{-i}{\sqrt{2}} \\
0 & 1 & 0 \\
\frac{-i}{\sqrt{2}} & -0 & \frac{1}{\sqrt{2}}
\end{array}\right) \\
& \mathbf{J}_{H W P}=\left(\begin{array}{ccc}
\frac{-i}{\sqrt{2}} & \frac{-i}{\sqrt{2}} & 0 \\
\frac{\sqrt{2}}{\sqrt{2}} & \frac{i}{\sqrt{2}} & 0 \\
0 & 0 & 1
\end{array}\right), \\
& \mathbf{J}_{N P B S}^{r}=\left(\begin{array}{ccc}
\frac{1}{\sqrt{2}} & 0 & 0 \\
0 & 0 & 1 \\
0 & -\frac{1}{\sqrt{2}} & 0
\end{array}\right) \text {, } \\
& \mathbf{J}_{N P B S}^{t}=\left(\begin{array}{ccc}
\frac{1}{\sqrt{2}} & 0 & 0 \\
0 & \frac{1}{\sqrt{2}} & 0 \\
0 & 0 & 1
\end{array}\right) \text {, } \\
& \mathbf{J}_{P B S}^{r}=\left(\begin{array}{lll}
1 & 0 & 0 \\
0 & 0 & 1 \\
0 & 0 & 0
\end{array}\right) \text {, } \\
& \mathbf{J}_{T I R}(\theta)=\left(\begin{array}{ccc}
1 & 0 & 0 \\
0 & \cos (2 \theta) & -2 \cos (\theta) \sin (\theta) \\
0 & -2 \cos (\theta) \sin (\theta) & -\cos (2 \theta)
\end{array}\right) \text {. } \\
& \mathbf{J}_{P B S}^{t}=\left(\begin{array}{lll}
0 & 0 & 0 \\
0 & 1 & 0 \\
0 & 0 & 1
\end{array}\right) \text {, }
\end{aligned}
$$

Where $\theta$ is the angle subtended between the light's wave-vector and the mirror surface normal vector. The polarization properties of a DoAP channel can be completely described by multiplying the PRT matrices of surfaces in the reverse order of propagation to form the 3D cumulative PRT matrix, 
P. The PRT matrices of the components of each channel is as follows:

$$
\begin{aligned}
& \mathbf{P}_{0^{\circ}}=\mathbf{J}_{P B S}^{t} \mathbf{J}_{Q W P} \mathbf{J}_{T I R}(-\pi / 2) \mathbf{J}_{Q W P} \mathbf{J}_{P B S}^{r} \mathbf{J}_{T I R}(-\pi / 4) \mathbf{J}_{N P B S}^{r} \quad=\left(\begin{array}{ccc}
0 & 0 & 0 \\
0 & 0 & -1 \\
-i 0.707 & 0 & 0
\end{array}\right) \\
& \mathbf{P}_{90^{\circ}}=\mathbf{J}_{T I R}(-3 \pi / 4) \mathbf{J}_{P B S}^{t} \mathbf{J}_{T I R}(-\pi / 4) \mathbf{J}_{N P B S}^{r} \quad=\left(\begin{array}{ccc}
0 & 0 & 0 \\
0 & 0 & -1 \\
0 & 0.707 & 0
\end{array}\right) \\
& \mathbf{P}_{45^{\circ}}=\mathbf{J}_{P B S}^{t} \mathbf{J}_{Q W P} \mathbf{J}_{T I R}(-\pi / 2) \mathbf{J}_{Q W P} \mathbf{J}_{P B S}^{r} \mathbf{J}_{H W P} \mathbf{J}_{N P B S}^{t} \quad=\left(\begin{array}{ccc}
0 & 0 & 0 \\
0 & 0 & -1 \\
-0.5 & -0.5 & 0
\end{array}\right) \\
& \mathbf{P}_{135^{\circ}}=\mathbf{J}_{T I R}(-3 \pi / 4) \mathbf{J}_{P B S}^{t} \mathbf{J}_{H W P} \mathbf{J}_{N P B S}^{t} \quad=\left(\begin{array}{ccc}
0 & 0 & 0 \\
0 & 0 & 0 \\
i 0.5 & -i 0.5 & 0 \\
-1
\end{array}\right)
\end{aligned}
$$

Equation 3 provides an example of how the cumulative PRT,

$$
\mathbf{P}_{\text {channel }} \cdot \mathbf{E}_{\text {in }}=\mathbf{E}_{\text {out }},
$$

relates the incident electric field, $\mathbf{E}_{i n}$, to the output electric field, $\mathbf{E}_{\text {out }}$. A transformation from 3D to Jones converts $\mathbf{P}$ into the local coordinate system by unfolding the optical path and assuming the wave-vector is parallel to the z-axis, to focus on the polarization properties of the system. A second matrix transformation converts the Jones matrix to a Mueller matrix. The analyzer vector for each path, relates the radiance exiting the DoAP to the input Stokes parameters. The analyzer vector is the first row in the path Mueller matrix. Table 1 lists the four analyzer vectors assuming ideal components in the DoAP.

Table 1 The PRT Mueller matrix for each of the DoAP output channels.

\begin{tabular}{|c|c|}
\hline DoAP channel & Mueller matrix \\
\hline \multirow{3}{*}{$\mathbf{0}^{\circ}$} & $\left(\begin{array}{cccc}0.25 & 0.25 & 0 & 0 \\
0.25 & 0.25 & 0 & 0 \\
0 & 0 & 0 & 0 \\
0 & 0 & 0 & 0\end{array}\right)$ \\
\hline \multirow{3}{*}{$\mathbf{9 0}^{\circ}$} & $\left(\begin{array}{cccc}0.25 & 0.25 & 0 & 0 \\
-0.25 & -0.25 & 0 & 0 \\
0 & 0 & 0 & 0 \\
0 & 0 & 0 & 0\end{array}\right)$ \\
\hline \multirow{4}{*}{$\mathbf{4 5}^{\circ}$} & $\left(\begin{array}{cccc}0.25 & 0.25 & 0 & 0 \\
0 & 0 & 0 & 0 \\
0.25 & 0.25 & 0 & 0 \\
0 & 0 & 0 & 0\end{array}\right)$ \\
\hline \multirow{3}{*}{$\mathbf{1 3 5}^{\circ}$} & $\left(\begin{array}{cccc}0.25 & 0.25 & 0 & 0 \\
0 & 0 & 0 & 0 \\
-0.25 & -0.25 & 0 & 0 \\
0 & 0 & 0 & 0\end{array}\right)$ \\
\hline
\end{tabular}

To construct the polarimetric measurement matrix, $\mathbf{P}_{M M}$, the analyzer vector from each of the four cumulative PRT matrices in 3 is transposed and concatenated into rows. Equation 4 shows the 
relationship of the polarimetric measurement matrix,

$$
\mathbf{P}_{M M} \mathbf{S}=\boldsymbol{\Phi},
$$

and incident Stokes vector, $\mathrm{S}$, to the flux vector, $\boldsymbol{\Phi}=\left[I_{0^{\circ}}, I_{90^{\circ}}, I_{45^{\circ}}, I_{135^{\circ}}\right]$, a list of flux measurements at the four output ports of the DoAP. Table 2 shows the transmitted flux for each DoAP channel in the ideal polarimeter for several incident polarization states, calculated using the ideal polarimetric measurement matrix.

Table 2 The relative output flux from each channel for a unit-intensity incident polarization state.

\begin{tabular}{|c|c|c|c|c|}
\hline Input polarization state & \multicolumn{4}{|c|}{ Output channel } \\
& $\mathbf{0}^{\circ}$ & $\mathbf{9 0}^{\circ}$ & $\mathbf{4 5}^{\circ}$ & $\mathbf{1 3 5}^{\circ}$ \\
\hline Unpolarized & 0.25 & 0.25 & 0.25 & 0.25 \\
\hline $\mathbf{0}^{\circ}$ & 0.50 & 0 & 0.25 & 0.25 \\
\hline $\mathbf{9 0}^{\circ}$ & 0 & 0.50 & 0.25 & 0.25 \\
\hline $\mathbf{4 5}^{\circ}$ & 0.25 & 0.25 & 0.50 & 0 \\
\hline $\mathbf{1 3 5}^{\circ}$ & 0.25 & 0.25 & 0 & 0.50 \\
\hline Right circular & 0.25 & 0.25 & 0.25 & 0.25 \\
\hline Left circular & 0.25 & 0.25 & 0.25 & 0.25 \\
\hline
\end{tabular}

For example, Table 2 shows the expected output fluxes for a horizontally $\left(0^{\circ}\right)$ polarized incident state are $(50 \%, 0 \%, 25 \%, 25 \%)$, which follows from the NPBS reflecting half of the light towards the $0^{\circ}$ and $90^{\circ}$ channels and transmitting half towards the $45^{\circ}$ and $135^{\circ}$ channels. The transmitted horizontally polarized light is rotated by the HWP and then split equally by the PBS between the $45^{\circ}$ and $135^{\circ}$ channels. The pseudo-inverse of matrix $\mathbf{P}_{\mathbf{M M}}$ is used to perform data reduction, operating on the flux vector, $\boldsymbol{\Phi}$, to calculate the linear Stokes parameters [S0, S1, S2], as described in PL\&OS, section 7.4.1. ${ }^{19}$

So the measured data is processed using the polarization data reduction matrix which is the pseudoinverse of the polarimetric measurement matrix. ${ }^{22}$ The analysis above describes an ideal polarimeter with ideal polarization elements. Real polarization elements, coatings, and optical components PRTs vary with wavelength and field. These can be evaluated with a polarization ray trace, such as Polaris-M, and a data reduction matrix constructed directly from polarization ray trace of the system. The detector measures radiance for each of the channels and the polarization data reduction matrix, $\mathbf{P}_{M M}^{-1} \boldsymbol{\Phi}=\mathbf{S}$, interprets these measurements to yield an estimate of the incident Stokes vector. The polarization data reduction matrix for the ideal case is shown in Eq. 5.

$$
\left[\begin{array}{cccc}
1 & 1 & 1 & 1 \\
2 & -2 & 0 & 0 \\
0 & 0 & 2 & -2
\end{array}\right] \cdot\left[\begin{array}{c}
P_{0^{\circ}} \\
P_{90^{\circ}} \\
P_{45^{\circ}} \\
P_{135^{\circ}}
\end{array}\right]=\left[\begin{array}{c}
I \\
Q \\
U
\end{array}\right]
$$

The ones in the first row of the polarization reduction matrix reconstructs the intensity value of the incident light. Terms in the second and third rows have opposite signs to find the difference in 
intensity measurements from orthogonal channels with a weight of 2 to compensate for the loss of signal caused by the NPBS. HiMAP's polarization reduction matrix is underdetermined, there are four equations and three unknowns with consistent linear equations, meaning there are mathematically

allowed solutions including null space components, according to the Rouché-Capelli theorem. ${ }^{23}$ In practice the pseudo-inverse $\mathbf{P}^{-1}$ of the polarimetric measurement matrix is used for data reduction to eliminate null space components, and thus noise, from the data reduction process. ${ }^{19}$ Once the Stokes vector has been reconstructed, the DoLP can then be calculated using Eq. 2 for all wavelengths and field positions.

\section{Tolerance Analysis Results}

\subsection{Waveplate Tolerance Analysis}

Parameterizing the polarization data reduction matrix from physical properties in the polarimeter enables an effective means for system modeling and tolerancing in preparation for alignment and calibration. Singular value decomposition of the polarimetric data reduction matrix yields the singular values, which is related to the square root of the system eigenvalues. ${ }^{24}$ The condition number is defined as the ratio of the smallest to the largest singular value and gives an indication of the sensitivity of the polarimeter to noise. A smaller condition number indicates a more stable, and thus noise resistant, data reduction. Thus the condition number is an indication of the ability to measure the DoLP and is an effective means to quantify design sensitivities. ${ }^{6}$ The condition number for HiMAP with ideal elements is $1 / \sqrt{2}$, and any deviations from this condition number indicate less accurate and desirable polarimeter designs. The effect of retardance and fast-axis orientation errors on the condition number is modeled by varying these parameters in different components of the DoAP, as shown in Table 3.

Table 3 The axial performance of the DoAP varies with small changes to component parameters.

\begin{tabular}{c|c|c}
\hline Parameter & Condition number & $\%$ Deviation from ideal \\
\hline $\pm 5^{\circ}$ QWP orientation & 1.41620 & $0.14 \%$ \\
$\pm 5^{\circ}$ QWP retardance & 1.41434 & $0.0085 \%$ \\
$\pm 5^{\circ}$ HWP orientation & 1.74345 & $23.0 \%$ \\
$\pm 5^{\circ}$ HWP retardance & 1.41556 & $0.09 \%$ \\
$\pm 22.5^{\circ}$ TIR retardance & 1.41421 & $0.0 \%$ \\
\hline
\end{tabular}

Parameters are individually perturbed to determine design sensitivities. For example, this condition number analysis reveals that the QWP orientation is more sensitive than the QWP retardance. Errors in QWP orientation will cause rotations of the s-state into elliptical states rather than the p-state, while variations to the QWP retardance mostly self-correct since these elements are double-passed (since they are attached to mirrors). The waveplate parameter with the greatest sensitivity is the HWP orientation which serves to rotate the $45^{\circ}$ and $135^{\circ}$ polarized states to s- and p-states, respectively. A $5^{\circ}$ misalignment in HWP orientation causes the $45^{\circ}$ and $135^{\circ}$ states to exit aligned at $10^{\circ}$ and $280^{\circ}$ respectively which then inhibits the ability of the PBS to completely separate these diagonal states. Errors to the HWP retardance have a lesser effect on the system, adding only a small amount 
of ellipticity to the exiting states. Minimal amounts of ellipticity can be mitigated by the doublepass PBS subsystem. Small changes to the HWP orientation however are exacerbated by the doublepass $\mathrm{PBS}$ subsystem as the $45^{\circ}$ and $135^{\circ}$ polarized states are no longer orthogonal. To limit the contribution of the half-wave plate orientation to a $1 \%$ condition number increase, the HWP fast-axis needs to be specified to within $\pm 0.3^{\circ}$. Thus the analysis of individual properties using this condition number technique enables designers to isolate design sensitivities, to establish a standard for baseline performance, and decide whether the expense of custom parts with tighter tolerances is justified. Using this introduction, the tolerance analysis of more complicated components, like thin film coating designs should now be easier to understand.

\subsection{Coating Tolerance Analysis}

A preliminary analysis of the sensitivity of the PBS and NPBS coating designs is performed by varying the reflection and transmission coefficients of the $\mathrm{s}$ - an $\mathrm{p}$ - states and calculating changes to the condition number.

HiMAP features two polarizing beamsplitters, each used to separate s- and p-polarized states. Typical specifications for extinction ratio are at least 50:1 for transmission and 10:1 for reflection over the 305-335 nm region, where ozone detection is most sensitive. The diattenuation of realistic PBS design varies with wavelength. Additionally, the wavelength response curve will undergo a red-shift with increasing angle of incidence (AOI) and blue-shift with decreasing AOI as the result of changes to the effective path length partial waves through thin film layers.

Specifications for a large AOI and bandwidth place strict conditions on the thin film designs. Polaris-M offers the ability to create a simple way to model and analyze various designs of the DoAP. A polarization ray tracing model is built using Polaris-M to perform analysis, integrating DoAP surfaces into a unified calculation of the condition number over wavelength and field. Thin film designs for the PBS and NPBS are each incorporated into Polaris-M to analyze their performance. Plots of the condition number over wavelength and field are shown in Fig. 4 for two coating designs under consideration. Analysis of the PBS shows that polarization state separation varies with AOI more so than wavelength. High order fluctuations seen in the left side of Fig. 4 (a) are common in broadband thin film polarizers and are the result of the discrete number of thin film layers used in the design of the PBS.

Analysis of the effect of the NPBS design on condition number is shown in Fig. 4 (b) showing that wavelength affects the performance more than AOI. An initial design of the NPBS was optimized for best RMS performance across the full bandwidth.

An ideal NPBS should exhibit no diattenuation, no retardance, and 50:50 flux splitting. The current working NPBS design consists of an aluminum layer sandwiched between glass layers. The metal layer helps to ensure non-polarizing beam splitting; however, an aluminum layer imparts some retardance and diattenuation. ${ }^{22}$ The reflected beam is analyzed for $0^{\circ}$ and $90^{\circ}$ polarized light and the transmitted beam is analyzed for $45^{\circ}$ and $135^{\circ}$ polarized light. Since Stokes parameters are constructed from orthogonal polarizations, small, polarization-invariant, differences in the reflectance and transmission of the NPBS have little effect on the condition number, and thus the system performance. 


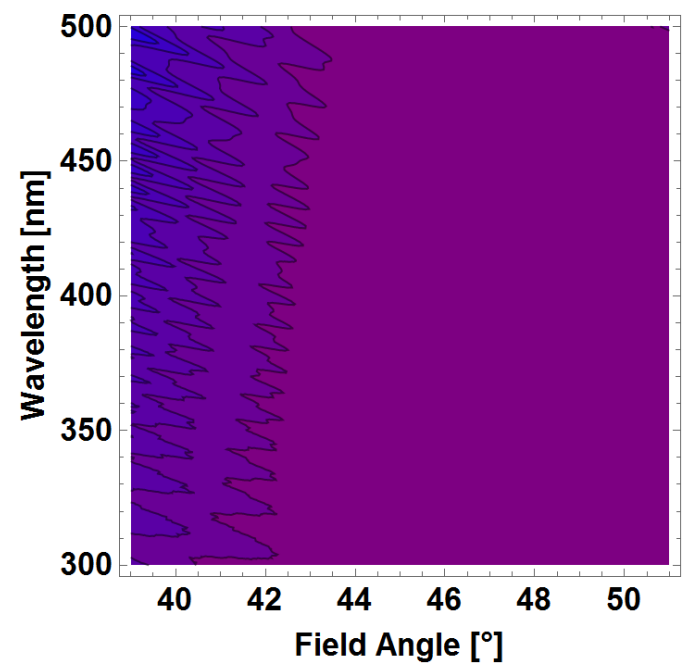

(a)

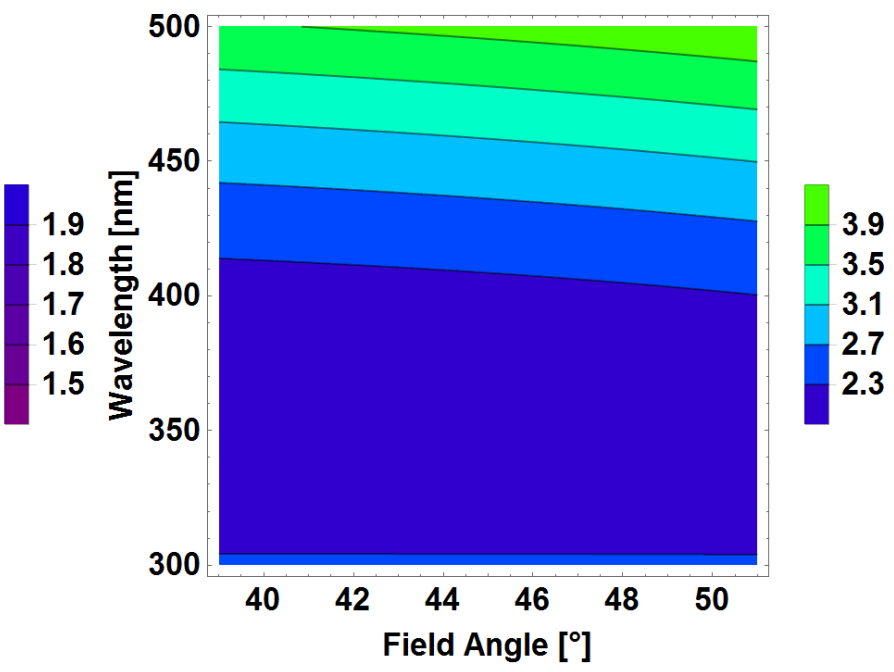

(b)

Fig 4 The condition number as a function of the wavelength and field angle as modeled with a PRT through a preliminary coating design of the (a) PBS and (b) NPBS.

\section{Conclusion}

This paper presents algorithms to tolerance polarimeters; specifically to calculate tolerances for waveplate retardances and orientations, polarizer extinction ratios, and thin film amplitude reflectance and transmission specifications. The polarimeter is described with 3D polarization raytracing calculus to tolerance individual polarization elements and their assemblies. The condition number of the polarimetric data reduction matrix is evaluated for non-ideal polarization elements and alignment errors. Application of these methods to HiMAP example shows that this polarimeter is very sensitive to the orientation of the half-wave plate fast-axis, while the quarter plates, which are double passed, have looser tolerances. Maintaining the half wave fast-axis within $\pm 0.3^{\circ}$ limits the increase of the condition number to $1 \%$.Such polarimetric tolerancing results provide a sound basis for specifying components for the DoAP.

These PRT algorithms can incorporate lenses, mirrors, gratings, and filters to show system performance variation with realistic tolerances providing a systematic means to quantify polarimeter performance and implement component tolerancing. 


\section{Acknowledgments}

The authors thank Angus MacLeod, Barry L. Lefer, Brian J. Drouin, Carl F. Ruoff, David Crisp, Eastwood Im, Glen Jaross, James P. Mcguire, John R. Worden, Michael R. Gunson, Pantazis Mouroulis, Parminder Ghuman, Pawan K. Bhartia, Randall R. Friedl, Randy Pollock, Richard S. Eckman, Robert O. Green, Simon J. Hook, Stanley P. Sander, Steven A. Macenka, Susan E. Owen, Thomas S. Pagano, and Vivienne H. Payne for many helpful discussions. Supports from the NASA ROSES-2013 Atmospheric Composition: Aura Science Team program (grant number: NNN13D455T), Jet Propulsion Laboratory (JPL) FY2018 Topic Area Research and Technology Development (R\&TD), and JPL Strategic Initiatives R\&TD programs gratefully acknowledged. 


\section{References}

1 "2014 nasa science plan,"

2 "2007 earth science decadal survey gacm,"

3 "Proceedings of a workshop held at nasa ames research center in may 2014.,"

4 "Global atmosphere composition mission,"

5 S. D. Ghude, C. Jena, D. Chate, et al., "Reductions in india's crop yield due to ozone," Geophysical Research Letters 41(15), 5685-5691 (2014).

6 J. S. Tyo, "Noise equalization in stokes parameter images obtained by use of variable-retardance polarimeters," Optics Letters 25(16), 1198-1200 (2000).

7 D. Fu, V. Natraj, T. Pongetti, et al., "Spectropolarimetric measurements of scattered sunlight in the huggins bands: Retrieval of tropospheric ozone profiles," (2011).

8 J. L. Neu, A. Al-Saadi, D. Edwards, et al., "Towards a next-generation global observing system for air quality: A white paper report to the 2017 decadal survey request for information," (2017).

9 K. Pickering, A. Thompson, M. Chin, et al., "Air quality in tropical and subtropical megacities: A white paper report to the 2017 earth sciences decadal survey request for information," (2017).

10 J. Worden, J. Neu, D. Jacob, et al., "Air quality impacts on crop and ecosystem health, a white paper submitted to the 2017 earth science decadal survey rfi \#1," (2017).

11 M. Lerdau, D. Schimel, and J. Neu, "Ozone impacts on crops and ecosystem health,” (2016).

12 D. Fu, J. R. Worden, X. Liu, et al., "Characterization of ozone profiles derived from aura tes and omi radiances," Atmospheric Chemistry and Physics 13(6), 3445-3462 (2013).

13 D. Fu, K. W. Bowman, H. M. Worden, et al., "High-resolution tropospheric carbon monoxide profiles retrieved from cris and tropomi," Atmospheric Measurement Techniques 9(6), 2567-2579 (2016).

14 D. Fu, S. S. Kulawik, K. Miyazaki, et al., "Retrievals of tropospheric ozone profiles from the synergism of airs and omi: methodology and validation," Atmospheric Measurement Techniques 11(10), 5587-5605 (2018).

15 H. Worden, D. Edwards, M. Deeter, et al., "Averaging kernel prediction from atmospheric and surface state parameters based on multiple regression for nadir-viewing satellite measurements of carbon monoxide and ozone," Atmospheric Measurement Techniques 6(7), 1633-1646 (2013).

16 K. Miyazaki, T. Sekiya, D. Fu, et al., "Balance of emission and dynamical controls on ozone during korus-aq from multi-constituent satellite data assimilation," Journal of Geophysical Research: Atmospheres (2018).

17 J. S. Tyo, D. L. Goldstein, D. B. Chenault, et al., "Review of passive imaging polarimetry for remote sensing applications," Applied optics 45(22), 5453-5469 (2006).

$18 \mathrm{~K}$. Twietmeyer and R. Chipman, "Condition number as a metric for the effectiveness of polarimetric algorithms," in Frontiers in Optics, FMB5, Optical Society of America (2005).

19 R. A. Chipman, W. S. T. Lam, and G. Young, Polarized Light and Optical Systems, CRC Press (2018).

20 R. A. Chipman and W.-S. T. Lam, "The polaris-m ray tracing program," in Polarization Science and Remote Sensing VII, 9613, 96130J, International Society for Optics and Photonics (2015).

21 A. Hessel, J. Schmoys, and D. Y. Tseng, "Bragg-angle blazing of diffraction gratings*," J. Opt. Soc. Am. 65, 380-384 (1975). 
22 K. Crabtree, "Polarization critical optical systems: Important effects and design techniques," (2007).

23 I. R. Shafarevich and A. O. Remizov, Linear algebra and geometry, Springer Science \& Business Media (2012).

24 G. H. Golub and C. F. Van Loan, Matrix computations, vol. 3, JHU Press (2012). 